\title{
In Defense of Lady Windermere Syndrome
}

\author{
Jerome M. Reich ${ }^{1,2}$ \\ Received: 13 March 2018 / Accepted: 4 May 2018 / Published online: 15 May 2018 \\ (c) Springer Science+Business Media, LLC, part of Springer Nature 2018
}

\begin{abstract}
Defense of Lady Windermere Syndrome (LWS) provides a critical analysis of its proposed pathogenesis, evidence supporting a causal role of volitional cough suppression, pathogenesis of M. avium complex (MAC) superimposition, a defense of the eponym, and cites a possible contribution of LWS to the bronchiectasis population.
\end{abstract}

Keywords Bronchiectasis $\cdot$ Cough $\cdot$ Lady Windermere Syndrome $\cdot$ M. aviumcomplex

$\begin{array}{ll}\text { Abbreviations } \\ \text { CF } & \text { Cystic fibrosis } \\ \text { CFTR } & \text { Cystic fibrosis transmembrane } \\ & \text { conductanceregulator } \\ \text { CTD } & \text { Connective tissue disorder } \\ \text { LWS } & \text { Lady Windermere Syndrome } \\ \text { MACPD } & \text { Pulmonary disease due to M. avium complex } \\ \text { PDPD } & \text { Predisposing pulmonarydisease } \\ \text { VCS } & \text { Volitional cough suppression }\end{array}$

Pulmonary disease due to M. avium complex (MACPD) occurs most often in White males with a predisposing pulmonary disease (PDPD), principally chronic obstructive pulmonary disease, healed tuberculosis and, particularly, bronchiectasis. It also affects individuals with cystic fibrosis (CF) and ciliary dyskinesia. Radiographically, it resembles disease due to M. tuberculosis with a proclivity to involve the upper lobes. Reich and Johnson reported on MACPD in 29 non-immunocompromised persons in a health maintenance organization, 6 (21\%) of whom lacked these characteristic features. The latter were exclusively older females lacking a PDPD whose involvement was confined to the lingula or middle lobe, principally the most peripheral portion [1]. Others treating MACPD repeatedly confirmed this distinctive constellation. Its pathogenesis is disputed. The authors proposed that volitional cough suppression (VCS) led to stasis of secretions in the affected regions creating a

Jerome M. Reich

Reichje@isp.com

1 Earle A. Chiles Research Institute, Portland, OR, USA

2 Portland, USA structural abnormality favoring MAC engraftment [1]. To convey this causative sequence, they assigned the eponym Lady Windermere Syndrome (LWS). Others suggested that a disorder of connective tissue or of airways clearance favoring MACPD underlies this syndrome. This editorial will critically examine the evidence for these views.

A proposed pathogenetic mechanism for LWS must account for all its features: female exclusivity, late age onset, susceptibility to MACPD absent a PDPD, and circumscribed involvement. Bronchiectasis, restricted to the middle lobe/ lingula, was uniformly evident on $\mathrm{CT}$ in these persons, and led to a search for its predisposing abnormalities. Contemporary proposed mechanisms include a CF form frustum, an unspecified connective tissue disorder (CTD) and VCS.

A CF variant would be expected to become evident early in life, not after a four-five-decade latency. In CF, the bronchiectasis predominantly affects upper lobes. [Because of the relative immobility of the upper thorax and its distance from the flailing movements of the ribs and diaphragm, these lobes (vs. lower lobes) have a disproportionate dependence for clearance of secretions on gravitational drainage vs. cough. The effectiveness of both is blunted in CF because of the defining viscidity and adherence of its bronchial secretions.] Some authors have advanced the concept that $\mathrm{CF}$ transmembrane conductance regulator (CFTR) gene variants, present in some individuals with LWS, signal its pathogenetic mechanism [2]. However, lacking reproduction of the CF phenotype, it is difficult to see how the presence of variant CFTR alleles could contribute to the pathogenesis of LWS. Moreover, Bergougnoux et al. reported no significant differences in the complement of CFTR variant alleles in persons with bronchiectasis vs. normals [3]. 
Several investigators have drawn attention to anatomic features shared by many individuals with LWS - tall stature, narrow chest AP diameter, pectus deformity, and mitral valve prolapse-as markers of a proposed, otherwise uncharacterized CTD predisposing to MACPD [4]. The presence of the first three would increase susceptibility by augmenting the requirement for vigorous cough to prevent stasis of secretions. Additionally, neither a CF variant nor a CTD would account for all the defining characteristics of LWS: advanced age of onset; gender exclusivity, distinctive localization, and MACPD susceptibility. Furthermore, both CF and CTD imply a genetic basis for an exclusively female entity, untenable for an X-linked disorder.

Volitional cough suppression In the initial report characterizing LWS, we inferred that the long, narrow, vertically oriented, segmental bronchi subserving the lingula and middle lobe, combined with the limited to absent collateral ventilation in these two regions, increased their dependence on cough for secretion clearance [1]. Tall stature and narrow anterior-posterior chest dimension magnify this regional susceptibility. Pectus excavatum, by compressing and elongating the lingula, can directly reduce clearance, potentiating the development of bronchiectasis. We hypothesized that VCS, more likely among fastidious females ("Ladies don't spit!"), led to stasis of secretions in these segments, and that secondary indolent infections resulted in regional bronchiectasis which predisposed to MACPD. (It is worth emphasizing that "cough" represents a spectrum extending from an ineffective, barely discernable rustling of air through a partially closed glottis to an explosive expulsion of air following vigorous thoracic and abdominal compression terminating in glottal abduction.) This deduction-based hypothesis furnished a plausible but unconfirmed accounting for the collective features of LWS. Later case reports in which the subjects acknowledged their habitual cough suppression furnished empirical verification [5]. Wells et al. coined the term "atussis nervosa" to draw a psychodynamic parallel to the volitional appetite suppression characterizing young women with anorexia nervosa and VCS in selected, otherwise healthy young women with bronchiectasis in whom tracheal air/fluid levels, evident on CT, furnished confirmation of its pathogenesis [6]. An array of disorders due to VCS has been identified, and it appears likely that its pathogenetic role is underappreciated [7].

Pathogenesis of MACPD in PDPD and LWS MAC is a congeries of saprophytic, free-living, soil- and water-dwelling bacteria, frequently present in municipal water supplies, where it can be aerosolized, e.g., by showers. It most frequently affects individuals with PDPD in which stasis of pooled (water containing) airway secretions is a shared characteristic. This unifying feature appears to be necessary and sufficient to account for colonization followed by invasion.
It adheres to Ockham's dictum, circumventing the need for additional hypotheses to account for susceptibility [8].

Defense of the eponym Several authors have criticized the trope, pointing to disparities between the stage character and the syndrome. The descriptor, LWS, was modeled after The Pickwickian Syndrome in which the nominal individual was Joe, the fat boy, who makes one brief appearance, not a Pickwick family member. It was not intended to reference a literal or figurative Lady Windermere. We selected the metaphorical eponym to provide an image, a visual "plectrum," to vividly convey the multiplicity of features characterizing the syndrome. We hoped that Oscar Wilde's choice of the character's name, combined with her title and British lineage, would convey an impression of fastidiousness. To criticize the trope on grounds that the fictional character was young did not literally suppress her cough or experience MACPD seems an exercise in aggravated pedantry, like urging the excision of the non-literal epithet, "lion hearted" from King Richard's name. To paraphrase Samuel Johnson: In literary ascriptions a man is not upon oath. The eponym LWS is more evocative, colorful, and succinct than the inelegant alternative, Nodular Bronchiectasis Phenotype of Pulmonary Disease Due to Nontuberculous Mycobacteria in Elderly Females of Obscure Genesis, and it has the apodictic advantage of acronymic brevity vs. NBPPDNTMEFOG.

Possible contribution of LWS MACPD in adult bronchiectasis Aksamit et al. reported advanced mean age, female predominance, and most frequent involvement of the middle lobe/lingula in persons with complicating non-tuberculous mycobacterial disease in their 13-institution study of non-CF bronchiectasis [9]. If a number of their older, female subjects exhibit isolated lingula/middle lobe involvement-the archetype of LWS - it would suggest that VCS is a material contributor to the bronchiectasis population, and that a remedy (Cough!) exists to diminish its occurrence.

\section{Compliance with Ethical Standards}

Conflict of interest The author declares no conflict of interest.

\section{References}

1. Reich JM, Johnson RE (1992) Mycobacterium avium-intracellulare disease presenting as an isolated middle lobe or lingular pattern: Lady Windermere syndrome. Chest 101:1605-1609

2. Olivier KN (2016) Lady windermere dissected: more form than fastidious. Ann Am Thorac Soc 13(10):1674-1676

3. Bergougnoux A et al (2015) Should diffuse bronchiectasis still be considered a CFTR-related disorder? J Cyst Fibros 4(5):646-653

4. Iseman MD, Buschman DL, Ackerson LM (1991) Pectus excavatum and scoliosis: thoracic abnormalities associated with pulmonary disease caused by Mycobacterium avium complex. Am Rev Respir Dis 144:914-916 
5. Dhillon SS, Watanakunakorn C (2000) Lady Windermere syndrome: middle lobe bronchiectasis and Mycobacterium avium complex infection due to voluntary cough suppression. Clin Infect Dis 30(3):572-575

6. Wells A, Rahman A, Woodhead M et al (1993) The clinical features of voluntary cough suppression associated with chronic pulmonary suppuration (atussis nervosa). A controlled evaluation. Am Rev Respir Dis 147:A461 (not published)
7. Reich JM (2014) Cough suppression disorders spectrum. Respir Med 108(2):413-415

8. McShane PJ, Glassroth J (2015) Pulmonary disease due to nontuberculous mycobacteria: current state and new insights. Chest 148(6):1517-1527

9. Aksamit TR, O'Donnell AE, Barker A et al (2017) Adult patients with bronchiectasis: a first look at the US bronchiectasis research registry. Chest 151(5):982-992 